\title{
Use of the Evolution RL Sheath During an Open-Heart Lead Extraction
}

Tsuyoshi Isawa, MD; Taku Honda, MD; Kazuhiro Yamaya, MD; Shigeru Toyoda, MD

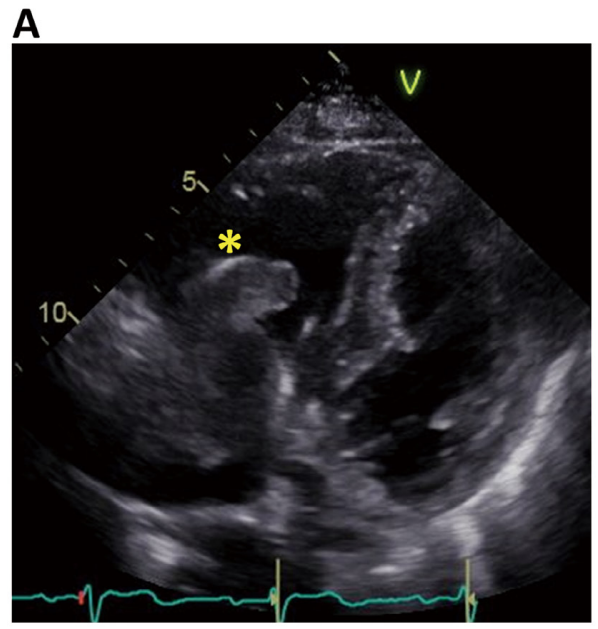

\section{B}

C
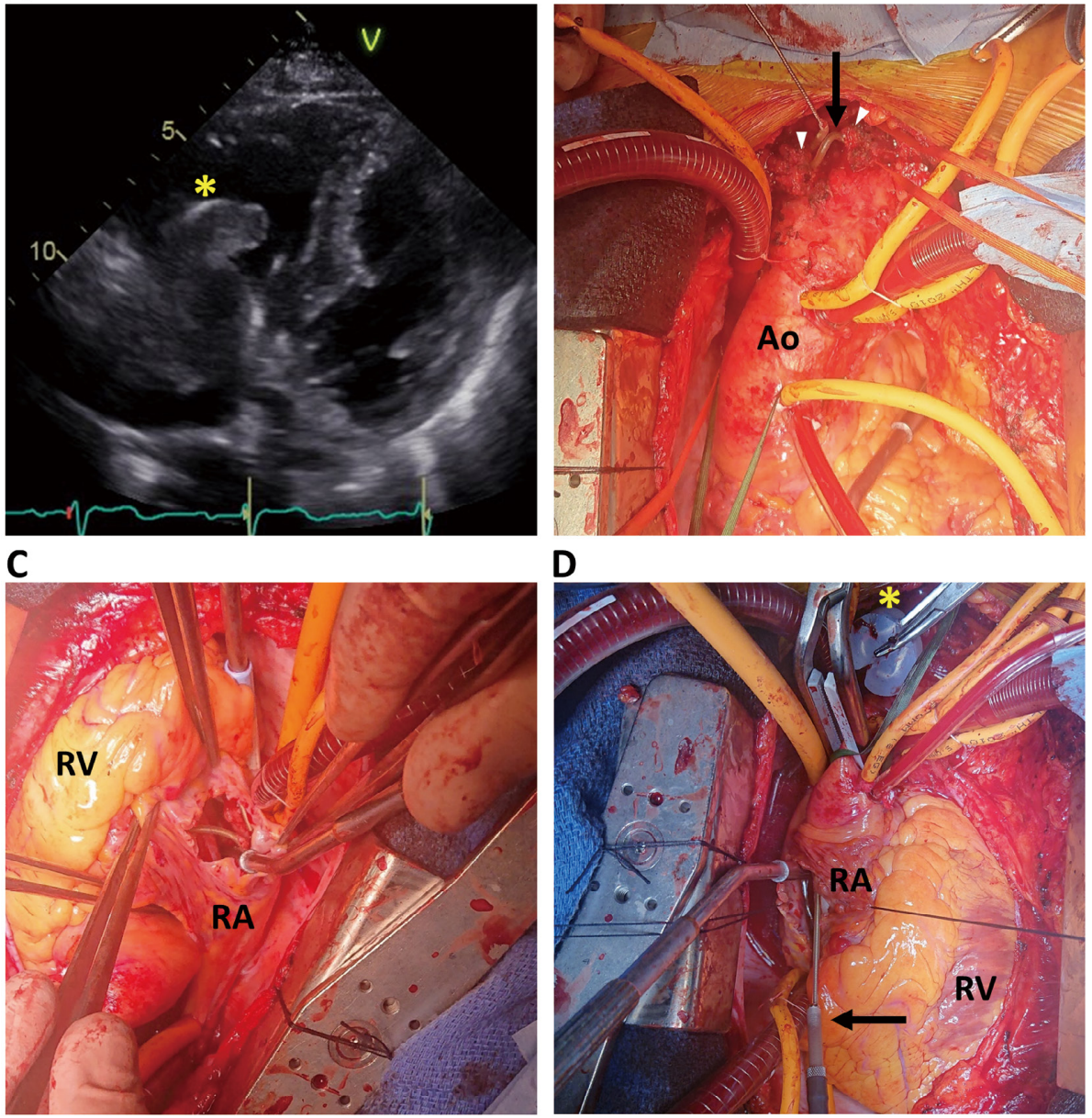

D

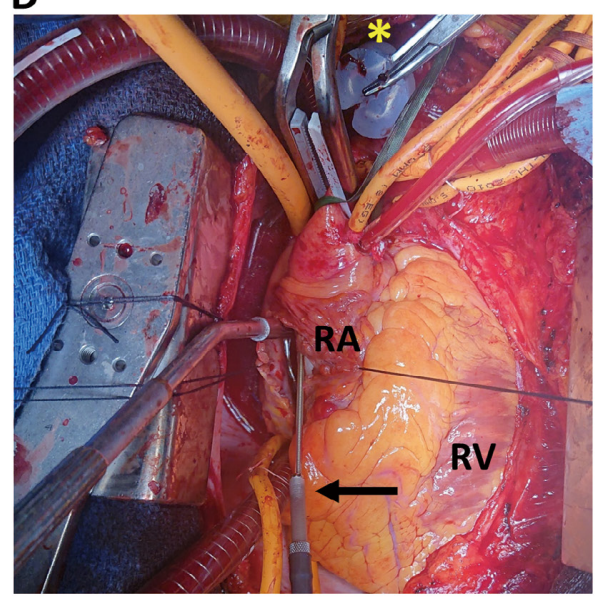

Figure. (A) Lead vegetation (asterisk). (B) The leads (arrow) were pulled out from the transected innominate vein (arrowheads). Ao, ascending aorta. (C) Separation of the lead from the atrial wall. RA, right atrium; RV, right ventricle. (D) Retrograde transatrial lead extraction: the tip of the lead was cut, and the inner lumen was intubated with a lead-locking device. Subsequently, a 9-Fr Evolution Shortie RL sheath (arrow) was advanced over the lead from the superior vena cava junction to the innominate vein while the lead was grasped with a Sof-Grip Hemostat (Cook Medical, Bloomington, IN, USA; asterisk).

Received August 1, 2021; accepted August 1, 2021; J-STAGE Advance Publication released online September 23, 2021 Time for primary review: 1 day

Department of Cardiology (T.I., T.H.), Department of Cardiovascular Surgery (K.Y.), Sendai Kousei Hospital, Sendai; Department of Cardiovascular Medicine, Dokkyo Medical University, Tochigi (S.T.), Japan

Mailing address: Tsuyoshi Isawa, MD, Department of Cardiology, Sendai Kousei Hospital, 4-15 Hirose-machi, Sendai 980-0873, Japan. E-mail: isa.tsuyo@gmail.com

All rights are reserved to the Japanese Circulation Society. For permissions, please e-mail: cr@j-circ.or.jp

ISSN-2434-0790

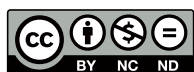


A

n 87-year-old man was referred to Sendai Kousei Hospital for lead extraction from an infected pacemaker. The patient had undergone dual-chamber pacemaker implantation in the left pectoral region 37 years ago. All leads were scheduled to be surgically extracted because lead vegetation of $>30 \mathrm{~mm}$ was identified (Figure A). Cardiopulmonary bypass was set up after a median sternotomy. The fibrotic adhesions around the leads in the occluded innominate vein were dissected and the leads were pulled out (Figure B). The tip of the lead was separated from the atrial wall (Figure C). After cutting the tip, the lead was intubated with a lead-locking device in a retrograde manner, followed by advancement of a 9-Fr Evolution Shortie RL sheath (Cook Medical, Bloomington, IN, USA; Figure D) from the superior vena cava junction to the innominate vein. In this manner, the atrial and ventricular leads were successfully extracted.

We believe that the Evolution RL sheath is more effective than a non-powered mechanical sheath for dissecting severely fibrotic adhesions, and its use as an adjunct during open-heart lead extraction may enable easier dissection of fibrotic adhesions around the leads.

None declared.

\section{Disclosures}

\section{IRB Information}

The study was approved by the Institutional Review Board of Sendai Kousei Hospital on July 14, 2021 (Approval no. 3-33). 